\title{
Dear Editor
}

The COVID-19 pandemic affects all of us, but consequences are more serious for those who are older or have chronic conditions and immune disorders. Places where numbers of people are living together, such as in nursing homes, have also proven to be particularly vulnerable. People with intellectual and developmental disabilities (I/DD) frequently present with the same risk factors but are largely forgotten in the care, response, and policy discussion.

Reports in New York are that people with I/DD have infections and death rates higher than the general population (Hakim, 2020). Although there is no evidence yet that having an I/DD, itself is a risk factor for COVID-19, well-documented health inequities (McCallion et al., 2017) for people with I/DD as they age are compounding risks:

- they have higher levels than others of multi-morbidity including heart and respiratory diseases and immune disorders;

- there are barriers they experience in accessing health care particularly as a group who have difficulty in describing symptoms; and

- historic stigma and views that their lives are less valuable pose additional risk, if decisions occur about who receives care when resources are scarce.

Many individuals with I/DD live with multiple people, and some do not understand the concept of keeping distance. Others have difficulty in reporting their symptoms, do not understand instructions for self-care, and experience imposed isolation and quarantining as punishing and bewildering. The places where people with I/DD live are not set up to support isolation spaces and, as was also noted by Hakim (2020), they have not been a priority for personal protective equipment (PPE) distribution. Finally, although the response of many service providers has been to "lock down" these settings with no visitors and no trips outside the home, there has necessarily been daily entry and exit of staff who themselves are largely untested, rarely have PPE and who may feel obligated to come to work even if unwell.

The service system was unprepared for COVID-19 at multiple levels and now needs to think about what will be different the next time. It needs the social workers in the system to not simply insist on change but to help build a pandemic/natural disaster responsive infrastructure.

When a person with I/DD is living at home, we have learned we need to be concerned about the risks for other family members, as well, particularly if the caregivers themselves are older and have their own chronic conditions. Future planning among persons with I/DD has been mostly focused upon transitions out of the family homes. Social workers, working in care management roles, must give equal attention to back-up plans, when caregivers becomes ill, and to advanced care planning for both the caregiver and the person with I/DD.

Individuals with I/DD regardless of where they live are falling through the cracks as frequently they are not a priority for testing or care-not because healthcare workers are uncaring, but because, as has been demonstrated in many communities, our systems and staff are overwhelmed. Social work needs to be at the forefront of creating disability friendly pathways to testing and care.

As rates of infection and of mortality appear to decline there is still a concern that there may be new "spikes" of infection, and potentially new waves of reoccurrence. For those with I/DD, service providers 
must radically respond by thinking about the person themselves, the individuals they live with, and protective strategies for the home. Training is needed for extra vigilance for symptoms for family members and staff working in settings with people who have I/DD. Procedures and strategies are needed for taking seriously any symptoms observed, rather than attributing such symptoms to disability. Attention should be geared to promoting activities that encourage staying home, handwashing, and keeping physical distance.

In isolation or quarantine, we all benefit from physical activity - one can find videos and come up with games, songs, and stories that include chair exercises or in-home physical activity. These activities also work for those who are caring for someone with I/DD. Pandemics/ natural disasters call upon us all to be our most creative in providing and managing these activities. Restricting our activities and staying in place will make many of us feel increasingly isolated. For people with I/DD, who often have smaller social groups and have fewer opportunities to find virtual alternatives, this loss of key connections will be felt even more.

Family and staff can help here, too. Social work attention is needed to planning and implementing alternative forms of contacts, including virtual so that they can be inclusive, anticipated, and enjoyed by those with disabilities, rather than missed. Perhaps most challenging is to have honest conversations about care when persons with I/DD experience serious and life-threatening symptoms. There has rightly been concern about the potential that in overwhelming situations such as in pandemics, care may be rationed and the criteria used may disadvantage people with disabilities. Social workers are already advocating to ensure that disability is not a criterion for exclusion (AASWSW, 2020). Decisions on whether to place people with I/DD in hospital, often unaccompanied, when infected, and/or to place them on ventilators in their last days also have their controversies. Social workers should be advocates that such decisions be guided by advanced care planning in which the persons with I/DD themselves have participated if possible.

Going forward, from this experience with a pandemic there are as well as practice and policy concerns, research needs for social work to address. These include documenting among people with I/DD the incidence and experience of COVID-19, successes and failures in offering support, and development and testing of interventions. The inclusion of people with I/DD and family members as co-researchers should be supported.

Philip McCallion School of Social Work, College of Public Health, Temple University, Philadelphia, Pennsylvania, USA

\section{References}

AASWSW. (2020). Policy of the American Academy of Social Work and Social Welfare (AASWSW) on the right of all Americans to potential life saving vaccinations and care.

Hakim, D. (2020, April 9). 'It's Hit Our Front Door': Homes for the Disabled See a Surge of Covid-19. New York Times. https://www.nytimes.com/2020/04/08/nyregion/coronavirus-disabilities-group-homes. html

McCallion, P., Jokinen, N., \& Janicki, M. P. (2017). Aging. In A comprehensive guide to intellectual and developmental disabilities, M.I. Wehmeyer, Brown, M., Percey, S. K., \& Fung, M. (Eds (Eds), Pp. 639- 654. MD: Paul Brookes Press: Baltimore. 